\title{
Eave ribbons treated with transfluthrin can protect both users and non-users against malaria vectors
}

\author{
Emmanuel P. Mwanga ${ }^{1 *} \mathbb{D}$, Arnold S. Mmbando ${ }^{1}$, Paul C. Mrosso ${ }^{1}$, Caleb Stica', Salum A. Mapua', \\ Marceline F. Finda 1,2 Khamis Kifungo ${ }^{1}$, Andrew Kafwenji ${ }^{1}$, April C. Monroe ${ }^{1,4,5,6}$, Sheila B. Ogoma', \\ Halfan S. Ngowo ${ }^{1,2}$ and Fredros O. Okumu ${ }^{1,2,3}$
}

\begin{abstract}
Background: Eave ribbons treated with spatial repellents effectively prevent human exposure to outdoor-biting and indoor-biting malaria mosquitoes, and could constitute a scalable and low-cost supplement to current interventions, such as insecticide-treated nets (ITNs). This study measured protection afforded by transfluthrin-treated eave ribbons to users (personal and communal protection) and non-users (only communal protection), and whether introducing mosquito traps as additional intervention influenced these benefits.

Methods: Five experimental huts were constructed inside a $110 \mathrm{~m}$ long, screened tunnel, in which 1000 Anopheles arabiensis were released nightly. Eave ribbons treated with $0.25 \mathrm{~g} / \mathrm{m}^{2}$ transfluthrin were fitted to $0,1,2,3,4$ or 5 huts, achieving $0,20,40,60,80$ and 100\% coverage, respectively. Volunteers sat near each hut and collected mosquitoes attempting to bite them from 6 to 10 p.m. (outdoor-biting), then went indoors to sleep under untreated bed nets, beside which CDC-light traps collected mosquitoes from 10 p.m. to 6 a.m. (indoor-biting). Caged mosquitoes kept inside the huts were monitored for $24 \mathrm{~h}$-mortality. Separately, eave ribbons, UV-LED mosquito traps (Mosclean) or both the ribbons and traps were fitted, each time leaving the central hut unfitted to represent non-user households and assess communal protection. Biting risk was measured concurrently in all huts, before and after introducing interventions.
\end{abstract}

Results: Transfluthrin-treated eave ribbons provided $83 \%$ and $62 \%$ protection indoors and outdoors respectively to users, plus $57 \%$ and $48 \%$ protection indoors and outdoors to the non-user. Protection for users remained constant, but protection for non-users increased with eave ribbons coverage, peaking once $80 \%$ of huts were fitted. Mortality of mosquitoes caged inside huts with eave ribbons was 100\%. The UV-LED traps increased indoor exposure to users and non-users, but marginally reduced outdoor-biting. Combining the traps and eave ribbons did not improve user protection relative to eave ribbons alone.

Conclusion: Transfluthrin-treated eave ribbons protect both users and non-users against malaria mosquitoes indoors and outdoors. The mosquito-killing property of transfluthrin can magnify the communal benefits by limiting unwanted diversion to non-users, but should be validated in field trials against pyrethroid-resistant vectors. Benefits of the UV-LED traps as an intervention alone or alongside eave ribbons were however undetectable in this study. These findings extend the evidence that transfluthrin-treated eave ribbons could complement ITNs.

Keywords: Eave ribbons, Ifakara Health Institute, Anopheles arabiensis, Malaria, Transfluthrin, Tanzania, Outdoor biting, Spatial repellents, Mosquito traps, Push-pull

\footnotetext{
*Correspondence: emwanga@ihi.or.tz

${ }^{1}$ Environmental Health and Ecological Sciences Department, Ifakara

Health Institute, Morogoro, Tanzania

Full list of author information is available at the end of the article
} 


\section{Background}

Vector control is the frontline malaria prevention strategy across Africa today. Insecticide-treated nets (ITNs) and house spraying with residual insecticides (IRS) alone contributed $81 \%$ of all gains accrued against malaria between 2000 and 2015 [1]. In Tanzania, effective vector control interventions contributed to a $50 \%$ reduction in malaria prevalence between 2008 and 2017 [2, 3], and the country is now pursuing an ambitious new strategy to reduce malaria prevalence below $1 \%$ by 2020 [4]. Today, ITNs and IRS continue to be the main interventions deployed against malaria vectors, however gaps in protection can remain due to various challenges, notably insecticide resistance [5], sub-optimal ITN access and use [6], high net attrition rates [7], and high levels of exposure to malaria vectors occurring outdoors or indoors before bed time $[8,9]$.

To complement these current strategies and advance efforts towards malaria elimination, complementary new tools are urgently required. In recent years, substantial effort has been put into creating an expanded vector control toolbox [10]. Some of these are techniques widely used in historical times with great success and are being brought back to the fore, such as larval source management [11] and mosquitoproof housing [12]. Others are the commodity-based approaches, such as insecticide-treated hammocks [13], insecticide-treated clothing [14], topical repellents [15], insecticides propagated by mosquitoes, i.e. auto dissemination [16], eave tubes combined with house screening [17], insecticide-treated livestock [18], entomopathogenic fungi [19], attractive targeted sugar baits [20], odour-baited mosquito traps [21], targeted spraying of mosquito swarms [22] and spatial repellent based technologies [23, 24]. Unfortunately, none of these so far have proven as scalable as ITNs in lowincome communities. Moreover, many still require high user compliance, which particularly hinders interventions such as topical repellents [25].

Spatial repellents are increasingly being proposed as a leading contender in the complementary vector control toolbox, especially since they can protect multiple persons at the same time and often function at sub-lethal doses [23]. Another advantage is that recent advances have demonstrated new delivery formats that require no electricity, remain effective over several months and have multiple modes of action against mosquitoes, including bite prevention, feeding inhibition and lethality [26-29]. In one example, hessian-based strips treated with transfluthrin prevented at least $75 \%$ of outdoor-biting by Anopheles arabiensis, Culex and Mansonia mosquitoes for at least 1 year [29]. Combinations of these spatial repellent products and odour-baited mosquito traps have also been evaluated, against indoor and outdoor-biting mosquitoes [30-33].

Mmbando et al. [32] recently described a related application, i.e. eave ribbons, which are simple hessian ribbons treated with spatial repellents and wrapped around eaves of houses (without completely blocking the eavespaces) to prevent outdoor-biting and indoor-biting mosquitoes over long periods of time. In the initial studies, they demonstrated high levels of protection in semi-field settings against laboratory-reared mosquitoes and also in experimental hut studies against field mosquitoes in rural Tanzania. It was concluded that this technology, pending further development and validation, has potential to complement current tools, as it is simple, low-cost, highly-scalable and easy-to-use; making it suitable even for poorly-constructed houses and low-income groups [31].

In a follow up study [31], Mmbando et al. evaluated different configurations of a push-pull system consisting of the transfluthrin-treated eave ribbons and odour-baited traps. Here, they concluded that efficacy of push-pull was mainly due to the spatial repellent component, and that adding odour-baited traps only slightly improved personal protection, but excessive trap densities increased exposure outdoors [31].

This current study addressed two important questions that remained unanswered regarding the eave ribbon technology. First, whether the eave ribbons at different intervention coverages, could effectively provide both personal and communal protection without diverting mosquitoes from users to non-users, as previously shown with transfluthrin-based mosquito coils under incomplete coverage situations [34]. Second, whether the personal and communal protection could be improved through different configurations of the push-pull systems, such as using indoor traps instead of outdoor traps as previously used [31], or placing the ribbons and traps in different houses instead of the same house as previously tested [30, 31, 35, 36]. For purposes of this study, a commercially-available UV-LED trap recently demonstrated as effective for trapping Tanzanian Anopheles and Culex mosquitoes was used as the intervention trap of choice alongside the eave ribbons [37].

\section{Methods \\ Mosquito tunnel and experimental huts}

This study was conducted inside a long, screened tunnel at Ifakara Health Institute's Mosquito City Facility in Tanzania (Fig. 1). This system is $110 \mathrm{~m}$ long, $3 \mathrm{~m}$ wide and $2.5 \mathrm{~m}$ high, and was designed to allow free movement of adult mosquitoes while interacting with hosts, houses or interventions. Often referred to as the Mosquito Marathon Tunnel, this system has previously been 
used for evaluating effects of distance and direction on mosquito responses to interventions such as attractants [38], odour-baited devices [39] or push-pull [32], as well as effects of new vector control technologies on densities and survival of mosquitoes $[39,40]$.

Five portable experimental huts $(3.0 \mathrm{~m}$ length $\times 2.7 \mathrm{~m}$ width $\times 2.0 \mathrm{~m}$ height) were constructed and positioned inside the tunnel, 20 meters away from each other. Four huts were regularly fitted with either transfluthrin treated eave ribbons or UV-LED mosquito traps as described below while one hut that neither had eave ribbons nor UV-LED mosquito traps acted as the control and was also used to measure effect to non-users.

\section{Eave ribbons and mosquito traps}

The eave ribbons were made with hessian fabric treated with $0.25 \mathrm{~g} / \mathrm{m}^{2}$ of transfluthrin as previously described by Mmbando et al. [31, 32]. This technology was originally designed for low-income households with gaps on eaves and walls. They are wrapped around open eaves without completely blocking the spaces, and are meant to protect from both indoor-biting and outdoor-biting mosquitoes. Previous versions of the eave ribbons were treated with higher doses of transfluthrin, and resulted in $>99 \%$ bite prevention indoors and outdoors. The $0.25 \mathrm{~g} / \mathrm{m}^{2}$ dose had initially provided $77 \%$ protection leaving enough protection gap for improvements. It was therefore selected for subsequent studies that evaluated the combination of the ribbons and traps in push-pull settings [31, $32]$. Once treated, hessian materials retain efficacy for 6 months years [29].

The selected trap was a new LED trap (Mosclean), which attracts mosquitoes by emitting $365 \mathrm{~nm}$ ultraviolet (UV) LED light and generates $\mathrm{CO}_{2}$ gas by photocatalytic reaction (Seoul-Viosys, Korea). The trap has recently been described in detail [37], and caught at least as many mosquitoes as human volunteers, or other previously used traps in semi-field and field tests [37]. Unlike in Mmbando et al. study which used odour-baited traps placed outdoors [31,32], the Mosclean trap, used in this current study was placed indoors. Both eave ribbons and traps are illustrated in Fig. 2.

\section{Mosquito releases and recaptures}

Laboratory-reared female Anopheles arabiensis were used, all obtained from colonies maintained at the Mosquito City facility. Each night, 1000 hungry adult females (3-5-days old) starved for $6 \mathrm{~h}$ by removing sugar meals from their cages, were released in the tunnel in batches of 250 between every two huts to ensure equal distribution throughout the tunnel.

Consenting adult male volunteers assisted in assessing biting risk outdoors and indoors as follows: one volunteer sat outdoors near each of the five huts and performed human landing catches (HLC) from 6 p.m. to 10 p.m. each night to trap mosquitoes attempting to bite them. The volunteers then moved inside the huts, slept under untreated bed nets and turned on a CDC-light trap placed next to the bed to catch host-seeking mosquitoes from 10 p.m. to 6 a.m. the next day. This procedure was first described in detail by Mmbando et al. [32], and enabled evaluation of both outdoor-biting risk (HLC catches) and indoor-biting risk (CDC-light trap catches) in ways generally representative of typical human-mosquito interactions in and around east African households $[8,9]$. These studies showed that most people are outdoors between 6 and 10 p.m. doing different household chores, before going indoors and eventually sleeping from $10 \mathrm{p} . \mathrm{m}$. to $6 \mathrm{a} . \mathrm{m}$. Though there were variations in actual times that people went indoors, we fixed the mosquito collection hours to the approximate the observed averages.

CDC-light traps and HLC were selected for being an effective and practical method for catching

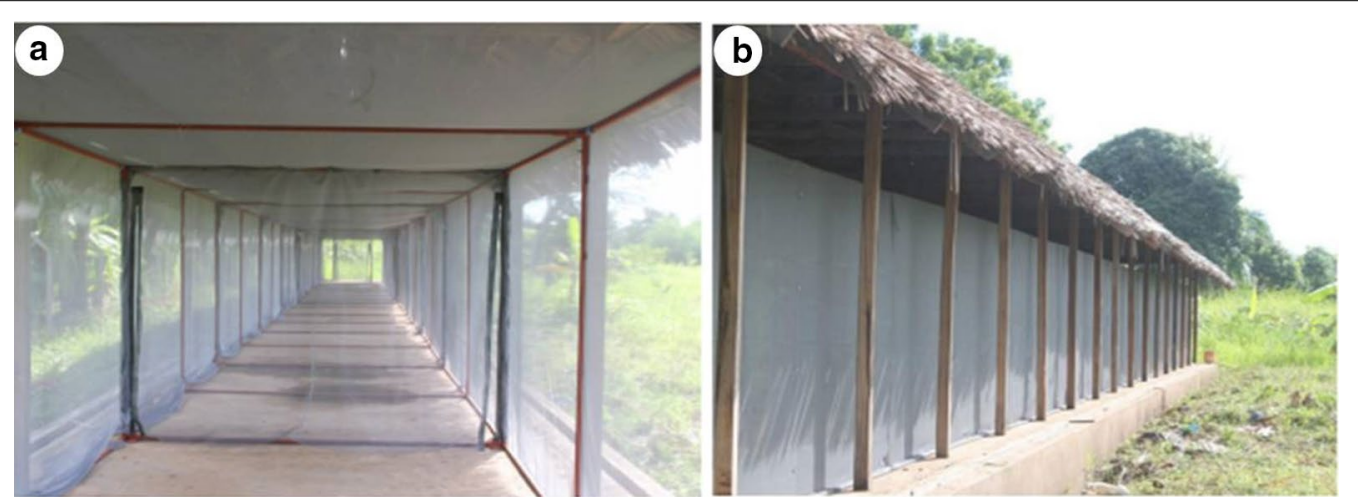

Fig. 1 Pictorial illustration showing the internal view of the long, screened tunnel when empty (a), and an outer view of the long screened tunnel (b) 


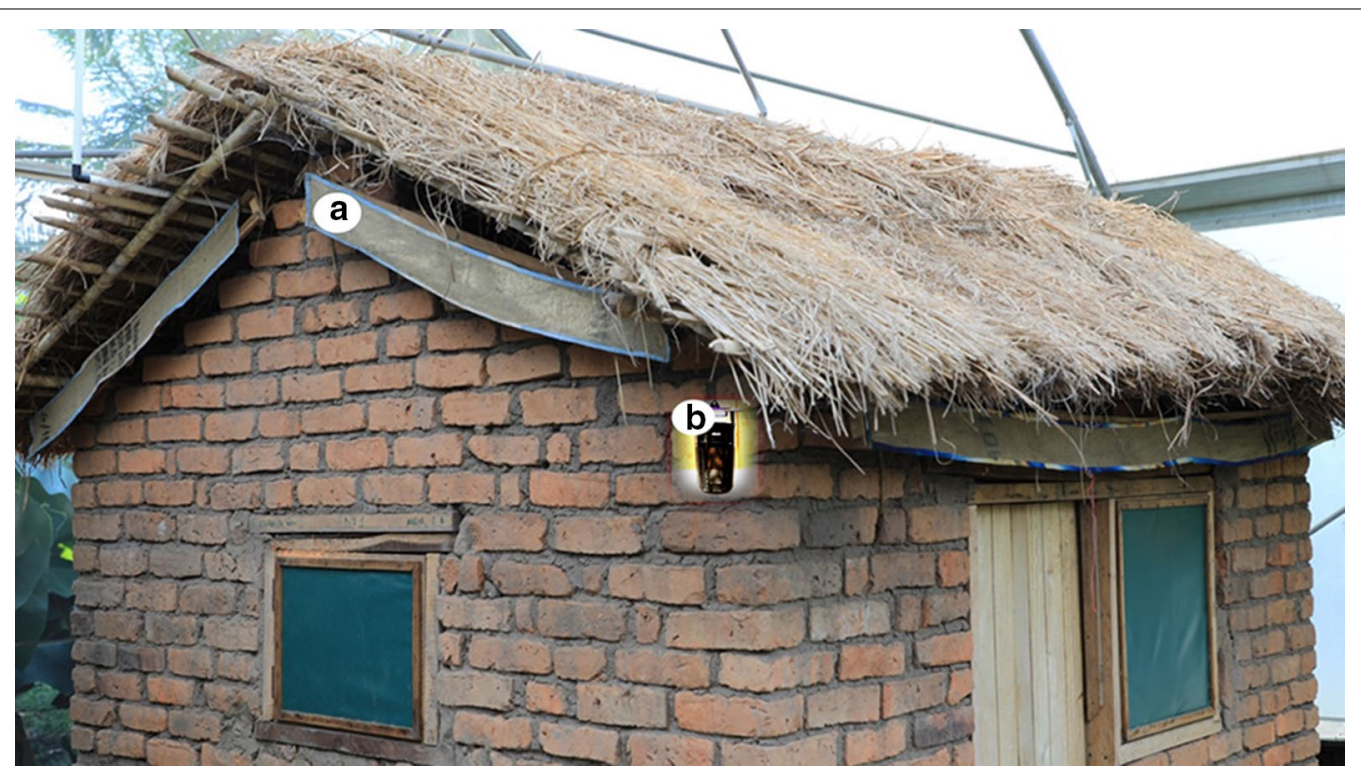

Fig. 2 Illustration of transfluthrin-treated eave ribbons wrapped around the eave space (a), and Mosclean trap (b). In this study, the Mosclean trap was used as an intervention and was placed always indoors. A separate indoor sampling trap, CDC-light trap was used for measuring efficacy of intervention. The eave ribbons and traps were assessed individually, or in push-pull system by putting them in the same houses or adjacent houses

Anopheles mosquito indoors and outdoors respectively. All huts were thoroughly cleaned and left unused for 2 days between different experiments or upon change of intervention, to minimize transfluthrin residual effects.

\section{Assessing protective efficacy afforded to users and non-users at different percentage coverages of transfluthrin-treated eave ribbons}

A baseline assessment of biting risk indoors and outdoors was first conducted for five nights with all the five huts having no intervention. Thereafter, protective efficacy was assessed at $20 \%, 40 \%, 60 \%, 80 \%$ and $100 \%$ coverage with transfluthrin-treated eave ribbons, each time for five consecutive nights. To assess efficacy at $20 \%$ coverage one of the five huts was randomly selected and fitted with the transfluthrin-treated eave ribbons, the remaining four huts being non-users. Next, two of the five huts were randomly selected and fitted with transfluthrin-treated eave ribbons, to achieve $40 \%$ coverage. Similarly, three, four or five huts were fitted with the treated eave ribbons to achieve $60 \%, 80 \%$ and $100 \%$ coverage respectively, all randomly assigned. Assessment of the protective efficacy in all five huts was done as described above. Based on estimates from previous experiments, these tests were conducted for a maximum of 30 nights, thus achieve satisfactory statistical power. Five nights were regarded as control (zero coverage) followed by 25 nights of treatment, five for each level of coverage. Each morning, all mosquitoes not recaptured were removed using electric aspirators.

\section{Assessing protective efficacy of transfluthrin-treated eave ribbons alone, Mosclean UV-LED traps alone, or combinations of the two interventions (i.e. push-pull) against indoor-biting and outdoor-biting risk among users and non-users}

The two different interventions were first evaluated individually in two separate experiments. All huts except the central non-user hut (also referred to us the sentinel hut) were fitted with either transfluthrin-treated eave ribbons alone (Fig. 3a), or Mosclean traps alone (Fig. 3b). Outdoor biting risk (from 6 to 10 p.m.) and indoor biting risk (from 10 p.m. to 6 a.m.) was measured as described above using HLC and CDC-light traps respectively. For each intervention, five nights pre-intervention period of mosquito collections was included to establish natural biting risk profile indoors and outdoors.

The third experiment was designed to assess potential of combining the push component (eave ribbons) and pull component (Mosclean traps) in mosaic format, where the two were not fitted to same hut as previously tested [31], but rather in separate huts. All huts except the sentinel hut were fitted with either transfluthrintreated eave ribbons alone (push) or the Mosclean trap alone (pull) as described in Fig. 3c, d. The sentinel hut always remained without either of the interventions and represented both a natural control and a non-user household. This push-pull mosaic experiment was done twice, first, when huts with eave ribbons were nearest to the non-user hut (Fig. 3c), and second when 
a

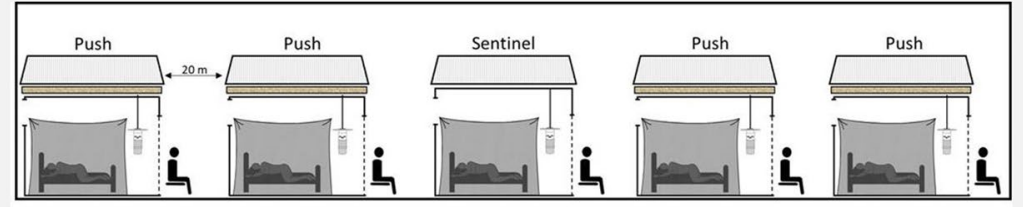

b
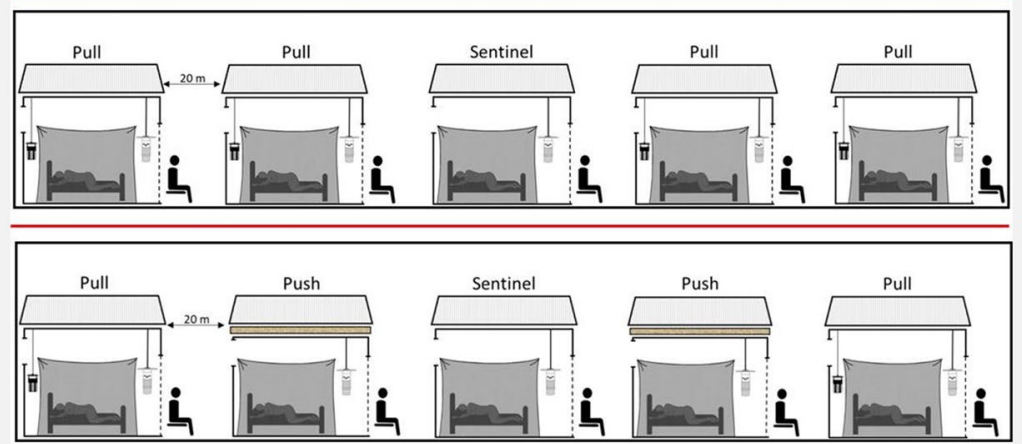

d

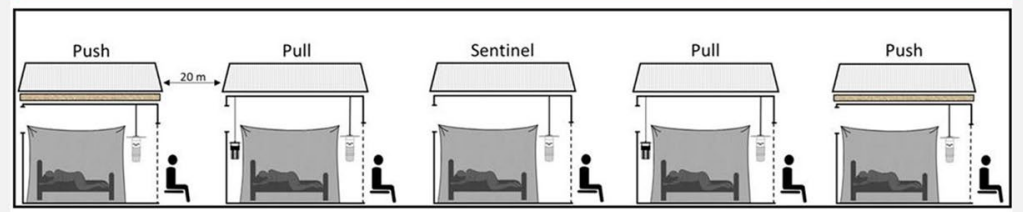

e

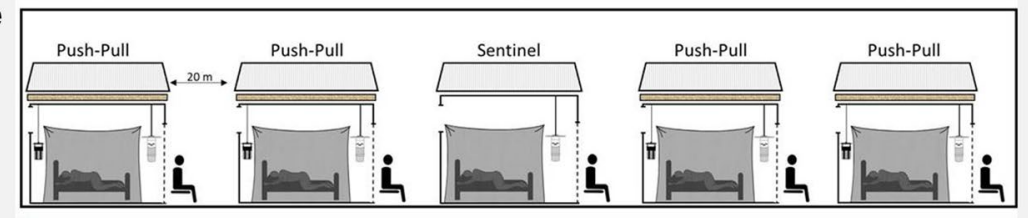

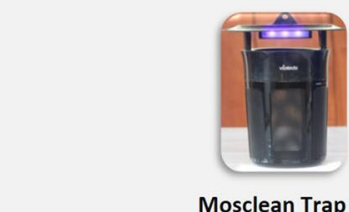

Mosclean Trap

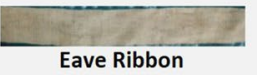

Eave Ribbon

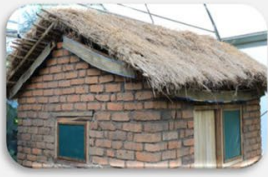

Experimental hut fitted with eave ribbons

$\underline{\text { Key }}$

Human landing catch outdoors

- CDC-light trap indoors

ii --.-- Mosclean trap indoors

ए-...- Transfluthrin-treated eave ribbons

Fig. 3 Illustration of the experimental setup to evaluate, protective efficacy of: push component only, i.e. all huts except non-user have transfluthrin-treated eave ribbons (a), and pull component only, i.e. all huts except non-user have Mosclean traps (b), push-pull mosaic, where the transfluthrin-treated eave ribbons, i.e. push, and Mosclean traps, i.e. pull are in different huts (c, $\mathbf{d})$, and standard push-pull system, where both eave ribbons and traps are in the same hut (e). Each night, adult consenting volunteers caught mosquitoes attempting to bite them outdoors from 6 to 10 p.m., then moved indoors under bed nets, beside which CDC-light traps caught mosquitoes from 10 to 6 a.m.

huts with Mosclean traps were nearest to the non-user hut (Fig. 3d). The experiments were each conducted for 15 consecutive nights, the first five nights being controls and the subsequent nights being intervention period.

Lastly, the efficacy of standard push-pull design, where all huts except sentinel are fitted with both traps and transfluthrin-treated eave ribbons was evaluated (Fig. 3e). Assessment of efficacy was conducted similar to the push-pull mosaic for 15 nights.

\section{Assessing mortality of mosquitoes exposed inside huts with or without transfluthrin-treated eave ribbons}

In this experiment, all huts except the sentinel hut were fitted with transfluthrin-treated eave ribbons. Netting cages, each containing 50 female An. arabiensis mosquitoes were suspended inside each of the five huts, 1.5 metres above ground, and left for $12 \mathrm{~h}$ overnight. The mosquitoes were then transferred to a separate room (average temp: $27 \pm 2{ }^{\circ} \mathrm{C}$; relative humidity: $80 \pm 5 \%$ ) and maintained on $10 \%$ glucose solution, then monitored for 24-h mortality. This experiment was also conducted for 15 nights. The first five nights were control nights, during which caged mosquitoes were introduced, but none of the huts had eave ribbons. This was followed by 10 nights during which mosquitoes were placed inside same huts, but this time four of the huts were fitted with transfluthrin-treated eave ribbons.

\section{Data analysis}

Data analysis was done using $\mathrm{R}$ statistical software, version 3.5.2 [41]. Comparisons of nightly collections were made between individual huts (treated and untreated) and also between periods before and periods after introducing the interventions. Number of 
mosquitoes collected indoors and outdoors, before and after treatment was compared by fitting Generalized Linear Mixed Models (GLMMs), using lme4 package [42]. Mean mosquito catches was first averaged for all the five huts for each of the five night pre-intervention periods to ascertain the baseline exposure levels indoors and outdoors.

To assess impact of interventions, mosquito counts were modelled following negative binomial distributions, with the main effect being treatment, i.e. whether huts had eave ribbons, traps or both, depending on experiment. Experimental nights and hut ID were included as random effects to account for variations between nights and huts. Protective efficacies, i.e. percentage protection accruable from the different interventions at the different huts, were calculated using model estimated means from pre-intervention and intervention nights using the formula, 100 * (Control-Treatment)/Control.

Any percentage protection observed in huts without the interventions was considered to represent community level protection affordable to non-users, while protection in houses with interventions was considered a combination of personal and community-level protection for users from interventions in their own huts and interventions in the neighboring huts. Graphical representations of the findings were created using $\mathrm{R}$ graphics package ggplot2 [43].

\section{Results}

Protective efficacy of transfluthrin-treated eave ribbons afforded to users and non-users at different intervention coverages

A summary of the findings is provided in Fig. 4. In comparison to control nights before introducing the eave ribbons, the number of mosquitoes caught indoors was reduced by more than $80 \%$ in all huts fitted with transfluthrin-treated eave ribbons. This percentage protection remained constant for users even when overall coverage increased from one of five huts $(20 \%)$ to five of five huts (100\%). Protection for users against outdoor exposures was $59 \%$ at $20 \%$ coverage, but slightly improved as coverage increased to a maximum of 69 protection at $60 \%$ coverage. Outdoor protection remained above $60 \%$ between $40 \%$ and $100 \%$ eave ribbon coverage.

For non-users, protection against indoor mosquito bites was low $(<20 \%)$ between 20 and $60 \%$ coverage levels, with modest $32 \%$ protection once eave ribbons coverage reached $80 \%$. Outdoor protection for non-users was modest (between 20 and $60 \%$ protection) but it increased to as high as $56 \%$ protection once intervention coverage reached $80 \%$.

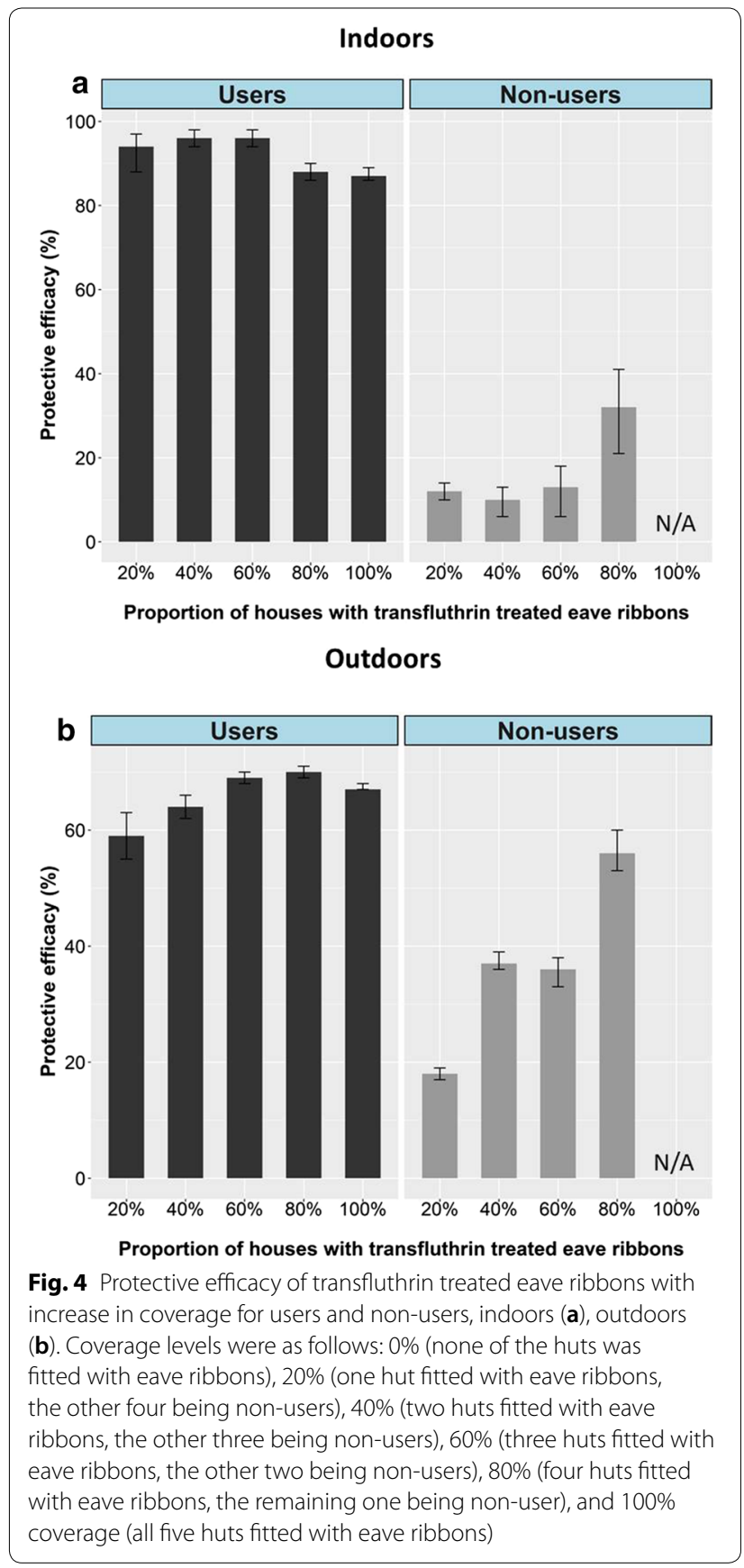

Protective efficacy of transfluthrin-treated eave ribbons alone, Mosclean traps alone, or combinations of the two interventions (i.e. push-pull) against indoor and outdoor-biting risk among users and non-users

Results of the different experimental designs including mean nightly catches in houses with and without the different interventions, and their associated protective efficacies are summarized in Table 1 and Figs. 5, 6. In the experiment where all huts except the sentinel 
hut were fitted with transfluthrin-treated eave ribbons, mosquito biting in the user huts was reduced by $83 \%$ indoors (Mean of 5.4 bites/night compared to 31.2 bites/night before intervention), and $62 \%$ outdoors (35.2 bites/night compared to 92.2 bites/night before intervention). Protection in the non-user hut was $57 \%$ indoors (14.6 bites/night compared to 34 bites/night before the intervention) and $48 \%$ outdoors (48 bites/night compared to 92 bites/night before the intervention).

In the experiment where all huts except the sentinel hut were fitted with Mosclean traps, mosquito densities indoors, as measured by CDC light traps increased by $38 \%$, but there was modest biting reduction of $25 \%$ outdoors. The same effect was observed in the non-user hut (the sentinel hut), where indoor densities increased by $52 \%$, and outdoor biting decreased by $10 \%$ (Table 1 and Fig. 6).

In the push-pull mosaic experiment, where eave ribbons and traps were used but in different huts, the huts with transfluthrin-treated eave ribbons received protection of $82 \%$ and $54 \%$ indoors and outdoors respectively. Huts with Mosclean trap received 28\% and 45\% protection indoors and outdoors respectively. The hut without intervention received only $9 \%$ protection indoors and 36\% protection outdoors (Table 1 and Fig. 6). Figures 5 and 6 also demonstrate that when the huts with Mosclean traps were near huts with transfluthrin-treated eave ribbons, the risk of mosquito bites was substantially lower than when all the intervention huts had Mosclean traps.

Lastly, in experiments evaluating the standard pushpull design, where the trap and spatial repellent were used in the same hut, users received $79 \%$ protection indoors and $50 \%$ protection outdoors. This approach however did not provide any protection to non-users indoors, but rather slightly increased exposure by $12 \%$. There was however modest protection to the non-user outdoors by $25 \%$.

\section{Number of mosquitoes caught by Mosclean traps}

The number of mosquitoes caught by Mosclean traps varied between the three different settings where they were used (Fig. 7). When Mosclean was used alone in the huts, it caught an average (and 95\% confidence interval) of 51.1 (46.0-56.9) mosquitoes per night. When used inside huts adjacent to other huts with eave ribbons, the catches were similar, i.e. 48.1 (43.2-53.5) per night. However, when this trap was used inside huts fitted with the transfluthrin-treated eave ribbons, the mean mosquito catches were reduced by half to just 22.1 (19.7-24.9) mosquitoes per night.

\section{Mortality of mosquitoes exposed inside huts with or without transfluthrin-treated eave ribbons}

The mortality rates in user huts and non-user huts are shown in Fig. 8, for the periods before introducing the intervention (i.e. control), and the period during which the transfluthrin-treated eave ribbons were in place (i.e. intervention). Average mortality of $100 \%$ was observed in mosquitoes held overnight inside huts with transfluthrintreated eave ribbons, compared to $4 \%$ mortality observed in the non-user hut. Mortality before introducing the ribbons was negligible in all huts.

\section{Discussion}

This study assessed whether transfluthrin-treated eave ribbons, previously demonstrated to protect against both indoor-biting and outdoor-biting mosquitoes in semifield and experimental hut studies [31, 32], could effectively protect both users and non-users without diverting mosquitoes from users to non-users. Another question addressed was whether the protection offered by these ribbons, either at personal or communal level could be improved by adding mosquito traps to form push-pull, and by different configurations of such push-pull systems. Two main differences in the configurations were as follows: (a) this current study used indoor intervention traps instead of outdoor traps as previously used [31], and (b) this study included one configuration with spatial repellents and traps in different houses and a another with both interventions placed in the same house as previously tested [30-33,36]. Overall, the studies lasted more than 450 hut nights, during which mosquito trapping was done indoors and outdoors, totalling more than 900 trap nights.

The most important finding was that transfluthrintreated eave ribbons can protect both users and nonusers. Besides, the protection afforded to the non-users peaked when the intervention was delivered at high coverages. In the tests where four of the five huts had the intervention, and the sentinel hut (i.e. non-user) did not, the eave ribbons provided $83 \%$ and $62 \%$ protection indoors and outdoors respectively to users, plus 57\% and $48 \%$ protection indoors and outdoors to the non-user. Separately, in the experiment where coverage was varied from 0 to $100 \%$, protection for users remained constant after $20 \%$ coverage while protection for non-users increased correspondingly with intervention coverage, peaking once $4 / 5(80 \%)$ of huts had received the eave ribbons. Protection for users was consistently higher indoors than outdoors, possibly due to: (1) surface area of the transfluthrin treated eave ribbons relative to the indoor space, (2) the preference of candidate vector species, An. arabiensis to bite more outdoors than indoors [44], (3) sub-lethal effect of transfluthrin, which may 


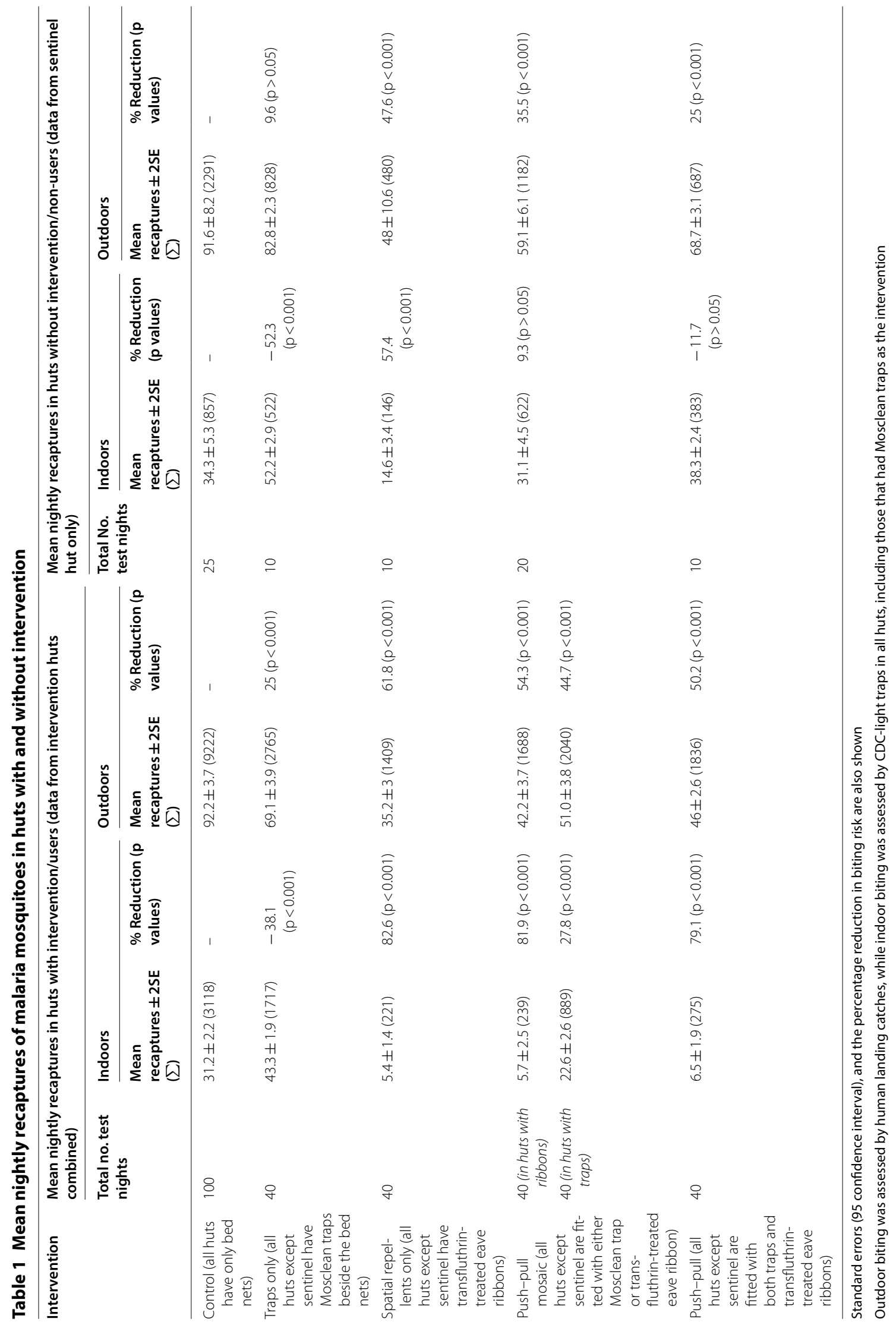



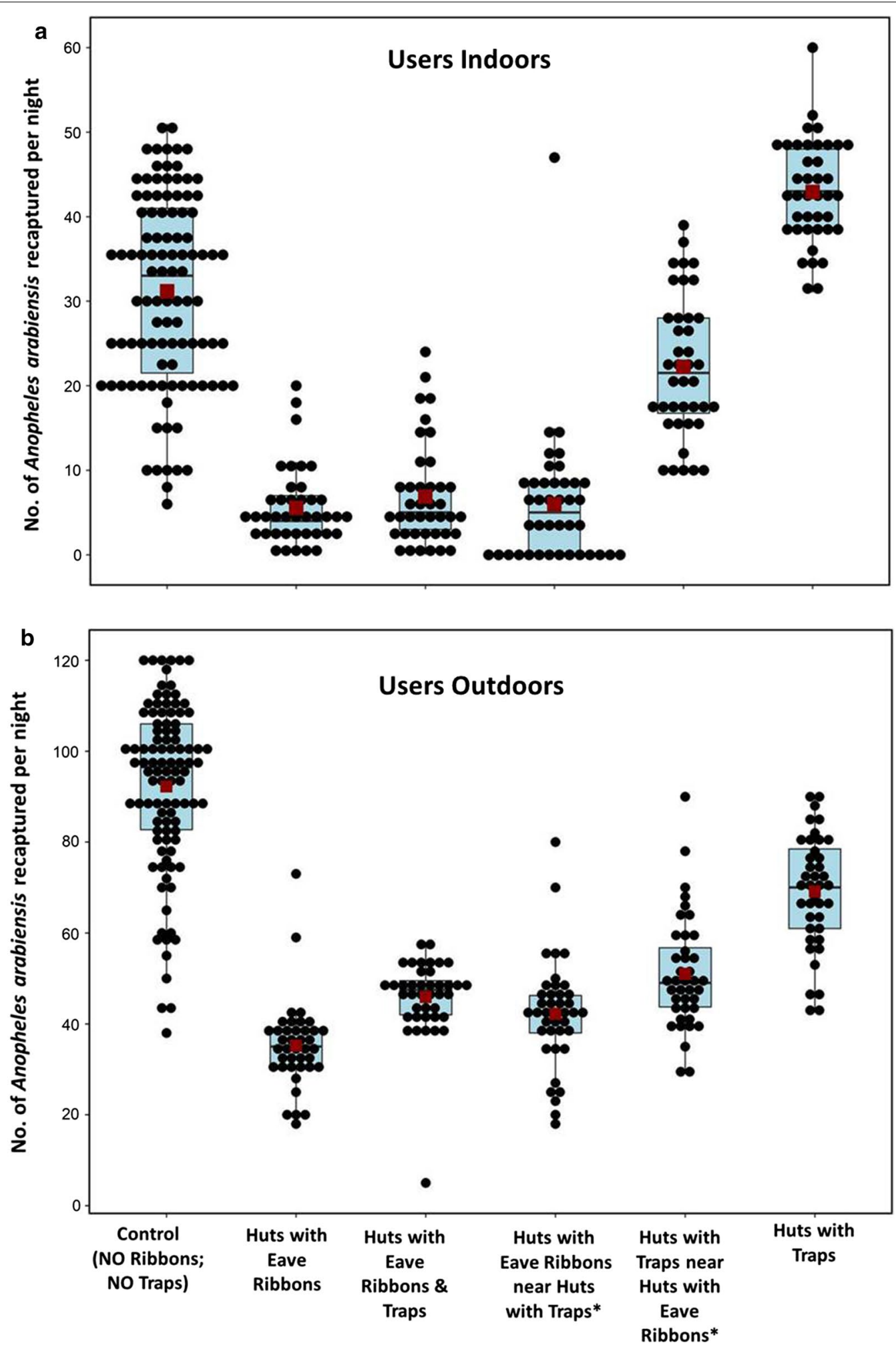

Fig. 5 Number of Anopheles arabiensis females recaptured per night indoors (a) and outdoors (b) at different huts of intervention users. Both median (black line crossing the box plot) and estimated means (red square inside the boxplot) are shown. Each black dot represents actual number of mosquitoes recaptured in different experimental nights. All the huts had intact untreated bed nets for basic protection. Observations were made before fitting traps or transfluthrin-treated eave ribbons (i.e. controls), and after the huts were fitted with either the eave ribbons (huts with eave ribbons), traps (huts with traps) or both eave ribbons and traps fitted to same hut (i.e. push pull). There were also observations, based on push-pull mosaic system, where half of the user huts had eave ribbons and the other half had traps $\left({ }^{*}\right)$ 

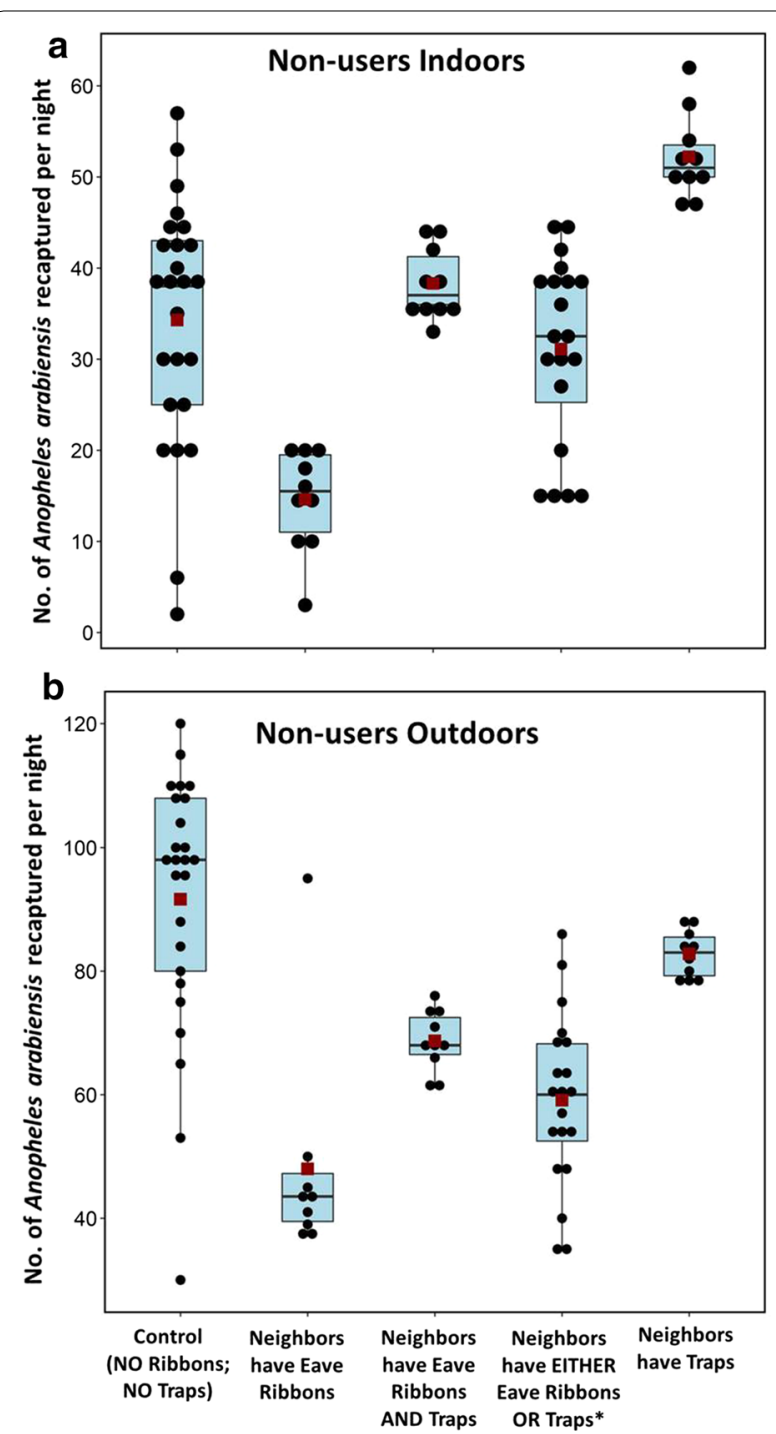

Fig. 6 Number of Anopheles arabiensis females recaptured per night indoors (a) and outdoors (b) at the hut of the non-user (i.e. the sentinel hut). Both median (black line crossing the box plot) and estimated means (red square inside the boxplot) are shown. Each black dot represents actual number of mosquitoes recaptured in different experimental nights. All the huts had intact untreated bed nets for basic protection. Observations were made at the sentinel non-user hut before fitting the neighboring huts with traps or transfluthrin-treated eave ribbons (i.e. controls), and then after the neighbors were fitted with either eave ribbons, traps or both traps and eave ribbons fitted to same hut. There was also observations based on push-pull mosaic system, where half of the neighbors huts had eave ribbons, and the other half had traps, i.e. Neighbors have EITHER traps OR eave ribbons (*)

disrupt host-seeking behaviour of mosquitoes entering the huts [28], and (4) the barrier effect of eave ribbons, however minimal [32]. The findings regarding personal protection to users corroborate those from the previous

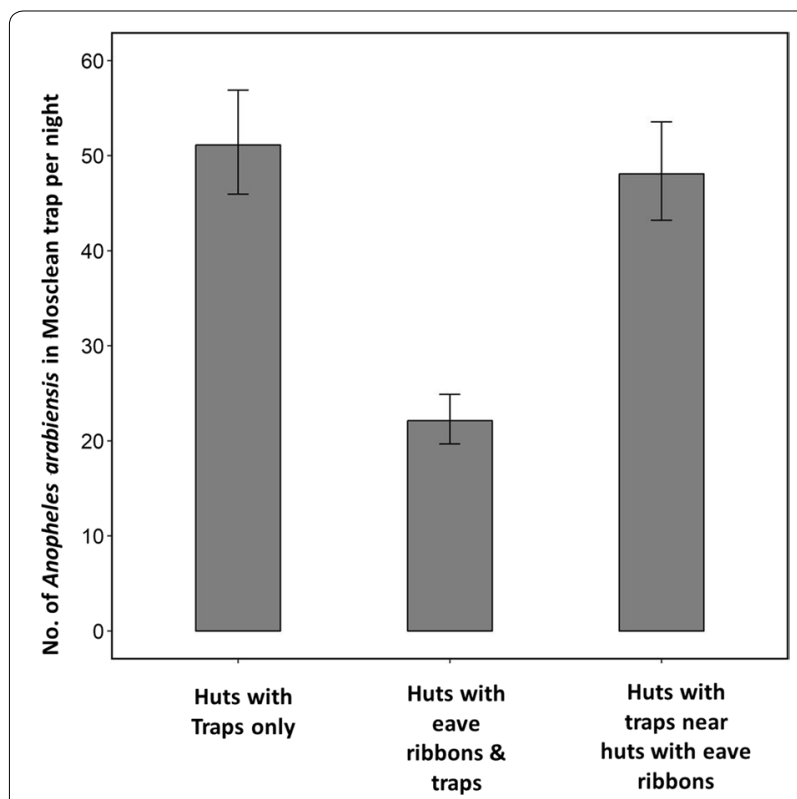

Fig. 7 Mean number of Anopheles arabiensis mosquitoes recaptured by CDC-light traps inside huts fitted with the UV-LED trap (Mosclean) alone or Mosclean trap and transfluthrin-treated eave ribbons.

Catches in huts with Mosclean Trap near huts with eave ribbons are also shown. The error bars represent the 95\% confidence intervals

studies which tested the efficacy of the transfluthrin treated eave-ribbons at both semi-field and field settings $[32,33]$. However, the findings relating to non-users represent the first report of such additional benefits associated with the eave ribbons technology.

An earlier study by Maia et al. [34] suggested that under conditions of incomplete coverage, transfluthrinbased products, in this case mosquito coils, provided personal protection to users, but could potentially divert mosquitoes to other users. In this current study, there was no increase in exposure to the non-users, possibly because the intervention also killed substantial proportions of the mosquitoes exposed in those huts, and which would have otherwise been diverted to the nonusers. Consistently, $100 \%$ of mosquitoes held inside the huts with transfluthrin-treated eave ribbons, died within $24 \mathrm{~h}$ of observation. Such levels of mortality indicate not only diminished likelihood of diversion of mosquitoes, but also suggest increased communal protection. However, it will be important to validate these findings in field settings where malaria mosquitoes are resistant to pyrethroids, and where such mortality may be reduced. Preliminary findings from rural south-eastern Tanzanian villages suggest that transfluthrin vapours remain substantially toxic to An. arabiensis, Anopheles funestus and Culex mosquito populations, which are known to be resistant against pyrethroids and carbamates (Mmbando 

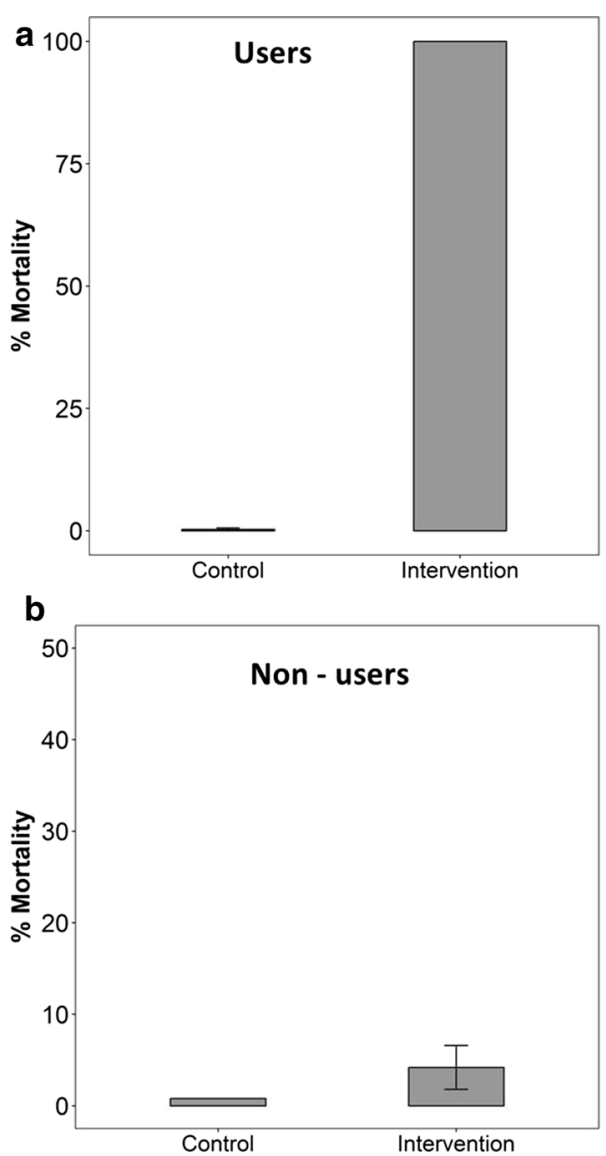

Fig. 8 Percentage mortality of mosquitoes exposed in huts with or without transfluthrin-treated eave ribbons, i.e. user huts (a) and non-user huts (b). The error bars represent the $95 \%$ confidence intervals

et al., unpublished). The first experimental hut study with eave ribbons in rural Tanzania also showed significant repellency against all these mosquito species despite being pyrethroid resistant. Nonetheless, this must be investigated in detail to guide future potential application of the eave ribbons technology.

These findings add to the body of evidence that spatial repellent eave ribbons can provide protection against malaria vectors both indoors and outdoors in the peridomestic space. The benefits extend beyond personal or household protection, and are accruable also non-users. Studies of night time human activities and sleeping patterns highlight the importance of interventions that can protect people during times when ITN use is not feasible, including when they are outdoors or indoors before going to sleep. Spending time outdoors near the home during evening hours, is common in many parts of subSaharan Africa [8], particularly in rural communities [8]. This includes activities such as cooking and other household chores, resting, and socializing in the hours before bed $[8,9,45]$. Spatial repellent eave ribbons offer a potentially viable solution for protecting people during these times and can offer protection to groups of people without requiring high levels of individual compliance.

Mosclean traps (pull component) alone resulted in increased risk of exposure to indoor mosquito bites in both the huts with taps and the sentinel hut without traps. Besides, it offered only minimal protection against mosquito bites outdoors. This trap, which has UV-LED and a photocatalytic $\mathrm{CO}_{2}$ generator as the main lures, was recently shown to be highly attractive and therefore effective for trapping mosquitoes indoors [37]. It could have therefore increased the overall densities indoors as measured by the CDC-light trap in the same huts. This also indicates that Mosclean may be attracting large densities of the mosquitoes to the vicinity, but it does not trap all the mosquitoes. In past experiments where $\mathrm{CO}_{2}$ baited traps were placed outdoors at the peri-domestic area, the traps provided only marginal protection against $A n$. arabiensis indoors, but increased biting risk to volunteers sitting outdoors [31]. An interesting observation however was that when the huts with the traps were near huts with transfluthrin-treated eave ribbons, the risk of mosquito bites was substantially lower than when all the huts had traps (Figs. 5, 6). The protective efficacy of the eave ribbons was, therefore, more obvious than that of the Mosclean traps at the scale of these studies. Mass trapping of mosquitoes generally has cumulative benefits, which could not be detected in this study since new batches of mosquitoes were released nightly.

Using the traps together with eave ribbons, whether in same houses or in different houses achieved less protection for non-users compared to eave ribbons alone (Table 1). For users, the protection achieved was similar to that obtained with just eave ribbons alone. The change from standard push-pull system to push-pull mosaic resulted in modest improvements in protection indoors or outdoors for the non-user, but these gains were still lower than what was achievable by transfluthrin-treated eave ribbons alone (Table 1). Moreover, in the push-pull mosaic design, the specific user huts that had Mosclean traps achieved lower percentage protection both indoors and outdoors than huts fitted with eave ribbons. The motivation for push-pull mosaic, as opposed to standard push-pull where huts are assigned both traps and repellents, had been to minimize any antagonistic effects between the two interventions at hut level, and also reduce costs and complexity of the overall application package. A related observation was that actual mosquito catches by the Mosclean traps themselves were halved upon introduction of eave ribbons, suggesting any value 
of these devices for removal trapping would be compromised by the spatial repellents.

Considering the complexities associated with using traps, especially those that require baits, Mmbando et al. [31] suggested that once high coverage with transfluthrin-treated eave ribbons is achieved, it may be best to not add traps at all. Nevertheless, since traps remove large numbers of mosquitoes from circulation, it is still theoretically possible that large-scale deployment may deliver communal level benefits overtime, as previously simulated by Okumu et al. [21]. Such cumulative impacts were also demonstrated in a trial of odour-baited trap in western Kenya, which achieved significant reductions in densities of An. funestus mosquitoes, and subsequently reduced malaria incidence by $40.8 \%$ and prevalence by $28.9 \%$ [46]. The potential benefits of traps, used alone or in combination with transfluthrin-treated eave ribbon, could however not be detected at the scale of this current study.

Most Anopheles traps have been developed for surveillance, and their applications as mass-trapping interventions remain circumstantial, often dependent on the type of lure used and the density of traps relative to human and animal hosts. In this study, Mosclean trap was selected and used as an intervention based on a previous study [37], where the trap was demonstrated to catch more mosquitoes than other commonly used traps such as CDC-light traps, BG-sentinel, and Suna traps.

One limitation of this study is that it was conducted in semi-field settings under controlled environment and using laboratory-reared mosquitoes. Second, the sentinel hut remained the same throughout the tests, and therefore lacked the natural variability associated with actual field settings. Lastly, the study used untreated mosquito nets for primary protection in all the huts instead of insecticide-treated bed nets (ITNs). The method for assessing exposure also did not consider the exposure to bites that occur when people are indoors but not yet under their nets. Instead the method used assumed that people went to bed as soon as they were indoors. Field validation of the findings may therefore be necessary, in different geographical locations, with different vector species composition. Preferably such studies should be in areas with pyrethroid resistant mosquitoes so that both the mortality and repellency effect, and any other effects of the intervention can be validated. An alternative validation strategy could be mathematical simulations, such as previously done for odour-baited traps [21]. Future research is needed to establish public health impact and inform successful execution of the technology.

\section{Conclusion}

Eave ribbons treated with protect both users and nonusers against malaria mosquitoes. The benefits to the non-users is highest where coverage of eave ribbons exceeds $80 \%$. Besides, most of the gains accrued by the non-users was in form of outdoor protection, even though percentage overall protection for both users and non-users was higher indoors. The mosquito-killing property of transfluthrin can magnify the communal benefits by limiting unwanted diversion to non-users, but should be validated in field trials against pyrethroidresistant vectors. The potential of the UV-LED traps as an intervention alone or alongside eave ribbons was however undetectable at the scale of this study. Overall, these findings extend the evidence that transfluthrin-treated eave ribbons could potentially complement ITNs. This study highlights the potential for eave ribbons to protect people in peri-domestic space before sleeping hours when ITN use is not feasible.

\section{Abbreviations \\ ITNs: insecticide-treated bed nets; UV-LED: ultraviolet-light emitting diodes; $\mathrm{CO}_{2}$ : carbon dioxide; $\mathrm{HLC}$ : human landing catch.}

\section{Acknowledgements}

We wish to convey special thanks to volunteers Mr. Said Nuru, Mr. George Simba, Mr. Odrick Mpira and Ramji Tweta for collection of mosquitoes in the semi-field experiments. We also express our deepest gratitude to the administrative support team at Ifakara Health Institute; particularly to Rukiyah M. Njalambaha for their assistance in study-related logistics.

\section{Authors' contributions}

EPM, MFF, PCM and FOO designed the study and developed research protocol. EPM, ASM, HSN and FOO analysed the data and wrote the manuscript. EPM, SAM, ASM, PCM, and HK conducted the experiments and data collection. CS designed all the schematics. FOO reviewed and revised the manuscript. The manuscript has been approved by all authors prior to submission. All authors read and approved the final manuscript.

\section{Funding}

This work was funded by Wellcome Trust Intermediate Fellowship in Public Health and Tropical Medicine Grant awarded to FO (Grant Number: WT102350/Z/13/Z). This research was also supported by the Howard Hughes Medical Institute (HHMI)-Gates International Research Scholarship awarded to FOO (Grant No. OPP1099295).

\section{Availability of data and materials \\ The dataset for this study is available from the corresponding author upon request.}

\section{Ethical considerations}

This study was approved by the Ifakara Health Institute Review board (Ref. IHI/IRB/EXT/No: 005-2018), and the Medical Research Coordinating Council at the Tanzanian National Institute of Medical Research (NIMR) (Ref. NIMR/ HQ/R.8c/Vol.II/880). Written and oral informed consent was obtained from all volunteers participating in this study, after being provided with all necessary information regarding goals, objective, benefits and risks associated with the study.

\section{Consent for publication}

Permission to publish the work was also obtained from NIMR (NIMR/HQ/P.12 VOL XXVIII/10). 


\section{Competing interests}

The authors declare that they have no competing interests.

\section{Author details}

${ }^{1}$ Environmental Health and Ecological Sciences Department, Ifakara Health Institute, Morogoro, Tanzania. ${ }^{2}$ Institute of Biodiversity, Animal Health and Comparative Medicine, University of Glasgow, Glasgow G12 8QQ, UK. ${ }^{3}$ School of Public Health, University of Witwatersrand, Johannesburg, South Africa. ${ }^{4}$ Johns Hopkins Center for Communication Programs, Baltimore, MD, USA. ${ }^{5}$ University of Basel, Basel, Switzerland. ${ }^{6}$ Swiss Tropical and Public Health Institute (Swiss TPH), Basel, Switzerland.

Received: 19 June 2019 Accepted: 11 September 2019 Published online: 18 September 2019

\section{References}

1. Bhatt S, Weiss DJ, Cameron E, Bisanzio D, Mappin B, Dalrymple U, et al. The effect of malaria control on Plasmodium falciparum in Africa between 2000 and 2015. Nature. 2015;526:207-11.

2. Tanzania Commission for AIDs, Zanzibar AIDS Commission, National Bureau of Statistics, Office of the Chief Government Statistician, Inc OMI. Tanzania HIV/AIDS and Malaria Indicator Survey, 2007-2008.

3. Ministry of Health Gender, Elderly and Children-MoHCDGEC/Tanzania community D, ICF. Tanzania Malaria Indicator Survey 2017_Final Report. 2018.

4. National Malaria Control Programme (Tanzania). Supplementary Malaria Midterm Strategic Plan (2018-2020). 2018.

5. Kisinza WN, Nkya TE, Kabula B, Overgaard HJ, Massue DJ, Mageni Z, et al. Multiple insecticide resistance in Anopheles gambiae from Tanzania: a major concern for malaria vector control. Malar J. 2017;16:439.

6. Mboma ZM, Overgaard HJ, Moore S, Bradley J, Moore J, Massue DJ, et al. Mosquito net coverage in years between mass distributions: a case study of Tanzania, 2013. Malar J. 2018:17:100.

7. Massue DJ, Moore SJ, Mageni ZD, Moore JD, Bradley J, Pigeon O, et al. Durability of Olyset campaign nets distributed between 2009 and 2011 in eight districts of Tanzania. Malar J. 2016;15:176.

8. Monroe A, Moore S, Koenker H, Lynch M, Ricotta E. Measuring and characterizing night time human behaviour as it relates to residual malaria transmission in sub-Saharan Africa: a review of the published literature. Malar J. 2019;18:6.

9. Finda MF, Moshi IR, Monroe A, Limwagu AJ, Nyoni AP, Swai JK, et al. Link ing human behaviours and malaria vector biting risk in south-eastern Tanzania. PLoS One. 2019;14:e0217414.

10. Williams YA, Tusting LS, Hocini S, Graves PM, Killeen GF, Kleinschmidt I, et al. Expanding the vector control toolbox for malaria elimination: a systematic review of the evidence. Adv Parasitol. 2018;99:345-79.

11. Tusting LS, Thwing J, Sinclair D, Fillinger U, Gimnig J, Bonner KE, et al. Mosquito larval source management for controlling malaria. Cochrane Database Syst Rev. 2013;8:CD008923.

12. Tusting LS, Bottomley C, Gibson H, Kleinschmidt I, Tatem AJ, Lindsay SW, et al. Housing improvements and malaria risk in sub-Saharan Africa: a multi-country analysis of survey data. PLoS Med. 2017;14(2):e1002234.

13. Thang ND, Erhart A, Speybroeck N, Xa NX, Thanh NN, Van Ky P, et al. Longlasting insecticidal hammocks for controlling forest malaria: a community-based trial in a rural area of Central Vietnam. PLoS One. 2009;4:e7369.

14. Kimani EW, Vulule JM, Kuria IW, Mugisha F. Use of insecticide-treated clothes for personal protection against malaria: a community trial. Malar J. 2006;5:63

15. Wilson AL, Chen-Hussey V, Logan JG, Lindsay SW. Are topical insect repellents effective against malaria in endemic populations? A systematic review and meta-analysis. Malar J. 2014;13:446.

16. Lwetoijera D, Kiware S, Okumu F, Devine GJ, Majambere S. Autodissemination of pyriproxyfen suppresses stable populations of Anopheles arabiensis under semi-controlled settings. Malar J. 2019;18:166.

17. Okumu F. The paradigm of eave tubes: scaling up house improvement and optimizing insecticide delivery against disease-transmitting mosquitoes. Malar J. 2017;16:207.
18. Rowland M, Durrani N, Kenward M, Mohammed N, Urahman H, Hewitt S. Control of malaria in Pakistan by applying deltamethrin insecticide to cattle: a community-randomised trial. Lancet. 2001;357:1837-41.

19. Scholte EJ, Nghabi K, Kihonda J, Takken W, Paaijmans KP, Abdulla S, et al. An entomopathogenic fungus for control of adult African malaria mosquitoes. Science. 2005;308:1641-2.

20. Müller GC, Beier JC, Traore SF, Toure MB, Traore MM, Bah S, et al. Successful field trial of attractive toxic sugar bait (ATSB) plant-spraying methods against malaria vectors in the Anopheles gambiae complex in Mali, West Africa. Malar J. 2010;9:210.

21. Okumu FO, Govella NJ, Moore SJ, Chitnis N, Killeen GF. Potential benefits, limitations and target product-profiles of odor-baited mosquito traps for malaria control in Africa. PLoS One. 2010;5:e11573.

22. Sawadogo SP, Niang A, Bilgo E, Millogo A, Maïga H, Dabire RK, et al. Targeting male mosquito swarms to control malaria vector density. PLoS One. 2017;12:e0173273.

23. Achee NL, Bangs MJ, Farlow R, Killeen GF, Lindsay S, Logan JG, et al. Spatial repellents: from discovery and development to evidence-based validation. Malar J. 2012;11:164.

24. Syafruddin D, Bangs MJ, Sidik D, Elyazar I, Asih PBS, Chan K, et al. Impact of a spatial repellent on malaria incidence in two villages in Sumba, Indonesia. Am J Trop Med Hyg. 2014;91:1079-87.

25. Gryseels C, Uk S, Sluydts V, Durnez L, Phoeuk P, Suon S, et al. Factors influencing the use of topical repellents: implications for the effectiveness of malaria elimination strategies. Sci Rep. 2015;5:16847.

26. Ogoma SB, Ngonyani H, Simfukwe ET, Mseka A, Moore J, Killeen GF. Spatial repellency of transfluthrin-treated hessian strips against laboratory-reared Anopheles arabiensis mosquitoes in a semi-field tunnel cage. Parasit Vectors. 2012;5:54

27. Masalu JP, Finda M, Okumu FO, Minja EG, Mmbando AS, Sikulu-Lord MT, et al. Efficacy and user acceptability of transfluthrin-treated sisal and hessian decorations for protecting against mosquito bites in outdoor bars. Parasit Vectors. 2017;10:197.

28. Ogoma SB, Ngonyani H, Simfukwe ET, Mseka A, Moore J, Maia MF, et al. The mode of action of spatial repellents and their impact on vectorial capacity of Anopheles gambiae sensu stricto. PLoS One. 2014;9:e110433.

29. Ogoma SB, Mmando AS, Swai JK, Horstmann S, Malone D, Killeen GF. A low technology emanator treated with the volatile pyrethroid transfluthrin confers long term protection against outdoor biting vectors of lymphatic filariasis, arboviruses and malaria. PLoS Negl Trop Dis. 2017;11:e0005455.

30. Menger DJ, Omusula P, Holdinga M, Homan T, Carreira AS, Vandendaele $P$, et al. Field evaluation of a push-pull system to reduce malaria transmission. PLoS One. 2015;10:e0123415.

31. Mmbando AS, Batista EPA, Kilalangongono M, Finda MF, Mwanga EP, Kaindoa EW, et al. Evaluation of a push-pull system consisting of transfluthrin-treated eave ribbons and odour-baited traps for control of indoor- and outdoor-biting malaria vectors. Malar J. 2019;18:87.

32. Mmbando AS, Ngowo H, Limwagu A, Kilalangongono M, Kifungo K, Okumu FO. Eave ribbons treated with the spatial repellent, transfluthrin, can effectively protect against indoor-biting and outdoorbiting malaria mosquitoes. Malar J. 2018;17:368.

33. Mmbando AS, Ngowo HS, Kilalangongono M, Abbas S, Matowo NS Moore SJ, et al. Small-scale field evaluation of push-pull system against early- and outdoor-biting malaria mosquitoes in an area of high pyrethroid resistance in Tanzania. Wellcome Open Res. 2017;2:112.

34. Maia MF, Kreppel K, Mbeyela E, Roman D, Mayagaya V, Lobo NF, et al. A crossover study to evaluate the diversion of malaria vectors in a community with incomplete coverage of spatial repellents in the Kilombero Valley, Tanzania. Parasit Vectors. 2016;9:451.

35. Menger DJ, Otieno B, De Rijk M, Mukabana WR, Van Loon JJ, Takken W. A push-pull system to reduce house entry of malaria mosquitoes. Malar J. 2014;13:119.

36. Menger DJ, Omusula P, Wouters K, Oketch C, Carreira AS, Durka M, et al. Eave screening and push-pull tactics to reduce house entry by vectors of Malaria. Am J Trop Med Hyg. 2016;94:868-78.

37. Mwanga EP, Ngowo HS, Mapua SA, Mmbando AS, Kaindoa EW, Kifungo $\mathrm{K}$, et al. Evaluation of an ultraviolet LED trap for catching Anopheles and Culex mosquitoes in south-eastern Tanzania. Parasit Vectors. 2019;12:418. 
38. Lorenz LM, Keane A, Moore JD, Munk CJ, Seeholzer L, Mseka A, et al. Taxis assays measure directional movement of mosquitoes to olfactory cues. Parasit Vectors. 2013;6:131.

39. Okumu F, Sumaye RD, Matowo NS, Mwangungulu SP, Kaindoa EW, Moshi $I R$, et al. Outdoor mosquito control using odour-baited devices: development and evaluation of a potential new strategy to complement indoor malaria prevention methods. Malaria World J. 2013:4:8.

40. Mmbando AS, Okumu FO, Mgando JP, Sumaye RD, Matowo NS, Madumla $E$, et al. Effects of a new outdoor mosquito control device, the mosquito landing box, on densities and survival of the malaria vector, Anopheles arabiensis, inside controlled semi-field settings. Malar J. 2015;14:494.

41. R Development Core Team. R: A Language and Environment for Statistical Computing. R Found Stat Comput Vienna Austria. 2018;0:\{ISBN\} 3-90005107-0. http://www.r-project.org/.

42. Bates $D$, Maechler $M$, Bolker B, Walker S. Fitting linear mixed-effects models using Ime4. J Stat Softw. 2015;67:1-48.

43. Wickham H. ggplot2. Springer-Verlag New York. 2009. http://link.springer. com/10.1007/978-0-387-98141-3.
44. Ngowo HS, Kaindoa EW, Matthiopoulos J, Ferguson HM, Okumu FO. Variations in household microclimate affect outdoor-biting behaviour of malaria vectors. Wellcome Open Res. 2017;2:102.

45. Monroe A, Asamoah O, Lam Y, Koenker H, Psychas P, Lynch M, et al. Outdoor-sleeping and other night-time activities in northern Ghana: implications for residual transmission and malaria prevention. Malar J. 2015;14:35.

46. Homan T, Hiscox A, Mweresa CK, Masiga D, Mukabana WR, Oria P, et al. The effect of mass mosquito trapping on malaria transmission and disease burden (SolarMal): a stepped-wedge cluster-randomised trial. Lancet. 2016;388:1193-201.

\section{Publisher's Note}

Springer Nature remains neutral with regard to jurisdictional claims in published maps and institutional affiliations.
Ready to submit your research? Choose BMC and benefit from:

- fast, convenient online submission

- thorough peer review by experienced researchers in your field

- rapid publication on acceptance

- support for research data, including large and complex data types

- gold Open Access which fosters wider collaboration and increased citations

- maximum visibility for your research: over $100 \mathrm{M}$ website views per year

At BMC, research is always in progress.

Learn more biomedcentral.com/submissions 